\title{
Exclusion of Giant Coronary Artery Aneurysm with Covered Stent Combined with Coil Embolization of Vessel Outflow
}

\author{
Puneeth Shridhar ${ }^{1}$, Triston Smith ${ }^{2}$, Ramzi Khalil ${ }^{3}$, David Lasorda ${ }^{3}$ and YoungJae Chun 1,4,5 \\ ${ }^{1}$ Department of Bioengineering, University of Pittsburgh, Pittsburgh, USA \\ ${ }^{2}$ Department of Cardiology, Wheeling Hospital, Wheeling, USA \\ ${ }^{3}$ Department of Cardiology, Allegheny General Hospital, Pittsburgh, USA \\ ${ }^{4}$ Department of Industrial Engineering, University of Pittsburgh, Pittsburgh, USA \\ ${ }^{5}$ McGowan Institute for Regenerative Medicine, Pittsburgh, USA
}

Corresponding author: Puneeth Shridhar, Department of Bioengineering, University of Pittsburgh, Pittsburgh, USA, Tel: +16502769952; E-mail: pus8@pitt.edu

Rec date: Jul 21, 2016; Acc date: Sep 27, 2016; Pub date: Sep 30, 2016

Citation: Shridhar P, Smith T, Khalil R, et al. Exclusion of Giant Coronary Artery Aneurysm with Covered Stent Combined with Coil Embolization of Vessel Outflow. Med Case Rep. 2016, 2:3.

\section{Abstract}

Background: Coronary artery aneurysms (CAA) are rare abnormal dilatation of the coronary arteries. They are termed giant if their diameter exceeds the reference vessel diameter by more than 4 times or if they are more than $8 \mathrm{~mm}$ in diameter. If not treated, they have a tendency to rupture, thrombose or embolize. There is however no definite therapy for CAA. Various therapies suggested in the management of aneurysms include surgical resection, covered stent implantation or coil embolization.

Methods: We describe a case of coiling of a potential collateral vessel feeding the giant coronary aneurysm retrogradely and covered stenting of main branch to exclude the aneurysm.

Results: Transcatheter embolization was successfully done. No late complications were seen during 1 year CT angiography.

Conclusion: Dual stent and coil embolization is safe and effective for vessel outflow during the treatment of giant coronary artery aneurysm. Further long term outcomes will have to be determined through serial CT angiographies.

Keywords: Giant aneurysm; Coronary artery; Covered stent; Embolization coil

\section{Introduction}

Coronary artery aneurysms (CAA) are abnormal dilatation of the coronary arteries measuring more than $50 \%$ of the reference vessel diameter [1].
There are various complications associated with coronary artery aneurysms which include thrombosis and distal embolization, rupture and vasospasm. The management of giant aneurysms mostly involves surgical resection [2], however this approach might be technically challenging and there are instances where the operating surgeons face difficulties in ligating the ends of the aneurysm, especially in presence of coexisting ventricular aneurysm [3].

We report a simultaneous coiling and stenting technique for treatment of a giant left circumflex aneurysm with vessel outflow.

\section{Case Report}

A 71-year-old man with history of coronary artery disease and ischemic cardiomyopathy underwent stenting of the first obtuse marginal (OM) artery with a drug eluting stent. Six months later, he developed a giant CAA at the site of the stent which on chest computed tomography angiography (CTA), it measured $4.0 \times 4.1 \mathrm{~cm}$. He subsequently underwent coronary artery bypass surgery with reverse saphenous vein (SVG) to $\mathrm{OM}$ artery along with the ligation of the distal end of the aneurysm. The proximal end of the aneurysm could not be ligated surgically. He also underwent repair of a $6 \mathrm{~cm}$ left ventricular aneurysm by endoventricular patch exclusion technique. During six month follow up, chest CTA showed that the aneurysm was persistent with dye filling into the aneurysmal sac hence the decision to percutaneously exclude the graft with a covered stent due to the risk of rupture. On coronary angiography, the SVG to OM was patent and a giant aneurysmal segment with a small outflow branch was noted in the proximal OM (Figures 1\&2).

Plan was made to perform coiling of the outflow branch followed by Jomed stent deployment into the left circumflex artery. FDA approval was sought to use Jomed stent under humanitarian device exemption. 


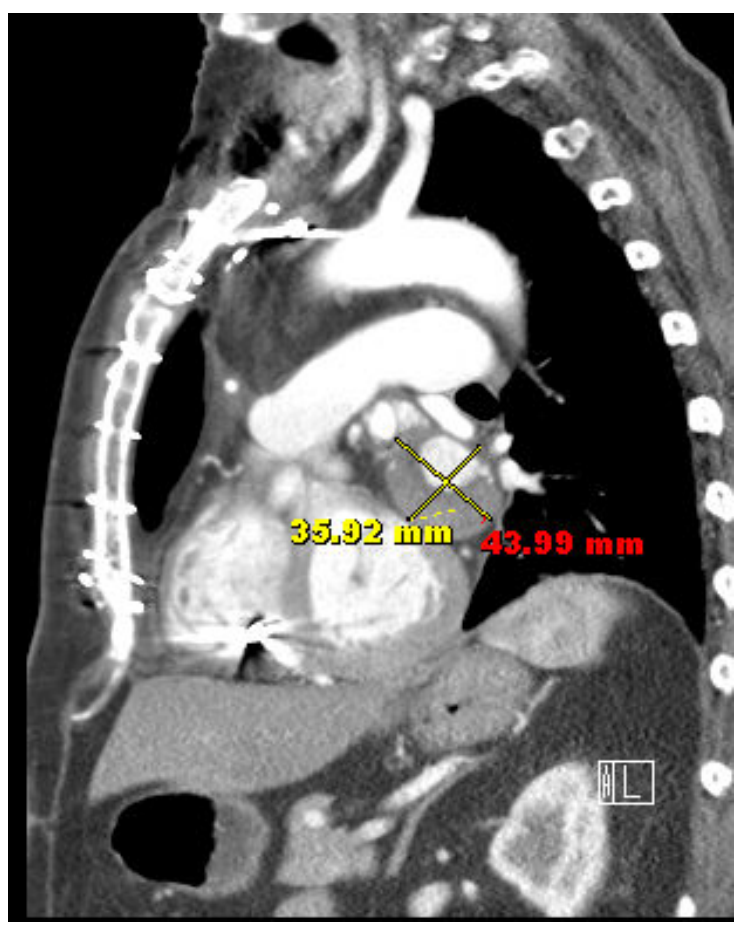

Figure 1 Contrast CT angiography of the chest before the procedure showing giant coronary aneurysm in the left circumflex artery.

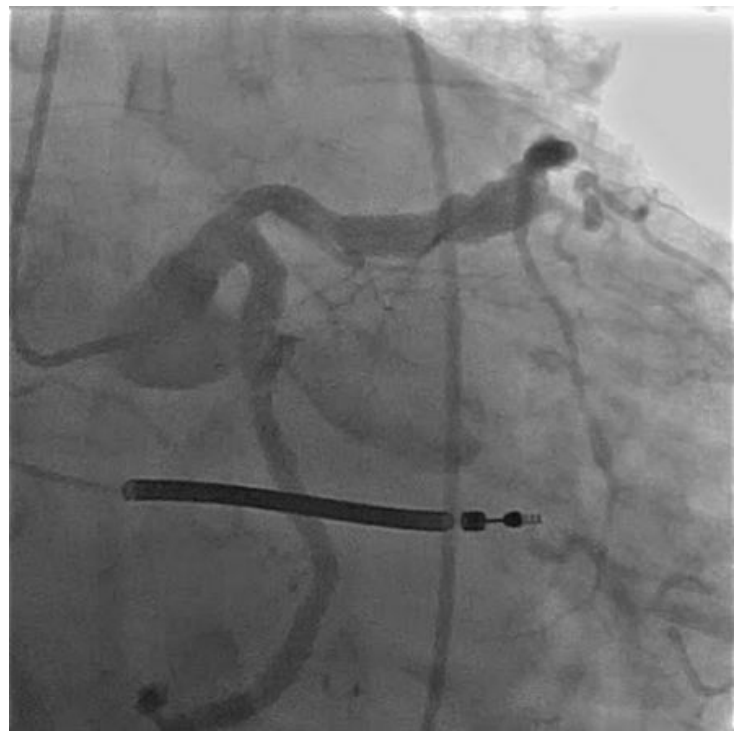

Figure 2 Angiographic image of the coronary aneurysm showing giant segment with small outflow branch of the proximal obtuse marginal artery.

Access was obtained through the right femoral artery using an 8 French sheath. We then engaged the vein graft to the OM using a Judkins Right 4 catheters (Infiniti Catheter, Johnson and Johnson/Cordis, Bridgewater, NJ) and XB 3.5 guiding catheter (Johnson and Johnson/Cordis, Bridgewater, NJ). On angiography, the vein graft to the obtuse marginal branch was found to be widely patent. The dominant circumflex artery had moderate ectasia with the proximal segment of the OM having a giant aneurysm. We advanced a $300 \mathrm{~cm} \mathrm{0.014"} \mathrm{HI} \mathrm{Torque}$ Extra S'port guide wire (Abbott Vascular Devices, Santa Clara, CA) into the aneurysm which was followed by $150 \mathrm{~cm}$ Renegade HighFlo microcatheter (Boston Scientific, Natick, MA). Subsequent injection of the dye through the Renegade catheter showed a small branch outflow of the aneurysmal dome. We coiled the feeding branch with three tornado coils (Cook, Bloomington, IN) and additional three Nester coils (Cook, Bloomington, IN) were deployed in the aneurysmal sac (Figure 3).

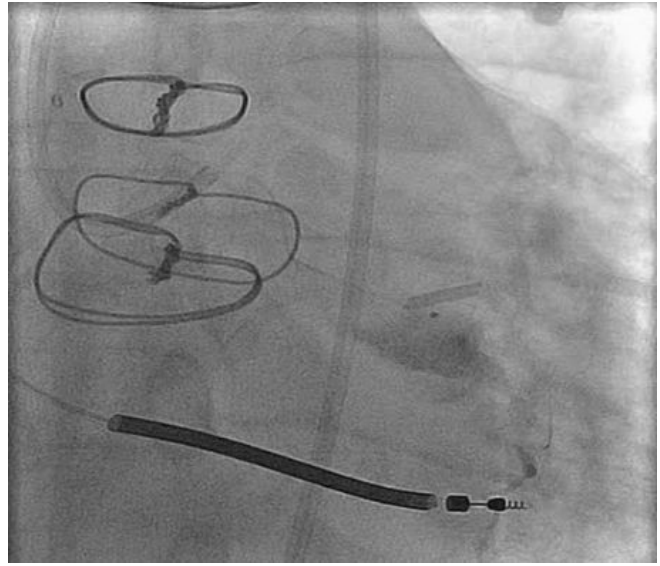

Figure 3 Angiographic image of the small outflow branch. Embolization coils can be seen deployed into feeding branch.

A guideliner catheter (Vascular Solutions Inc, Minneapolis, MN) was advanced into the sac to achieve better support. The sac angiography confirmed that the outflow branch was adequately coiled. A $4.0 \times 19 \mathrm{~mm}$ Graftmaster Jostent (Abbott Vascular Devices, Santa Clara, CA) was advanced over a $190 \mathrm{~cm}$ $\mathrm{HI}$ Torque S'port wire. The stent was deployed at 20 atms under fluoroscopic guidance.

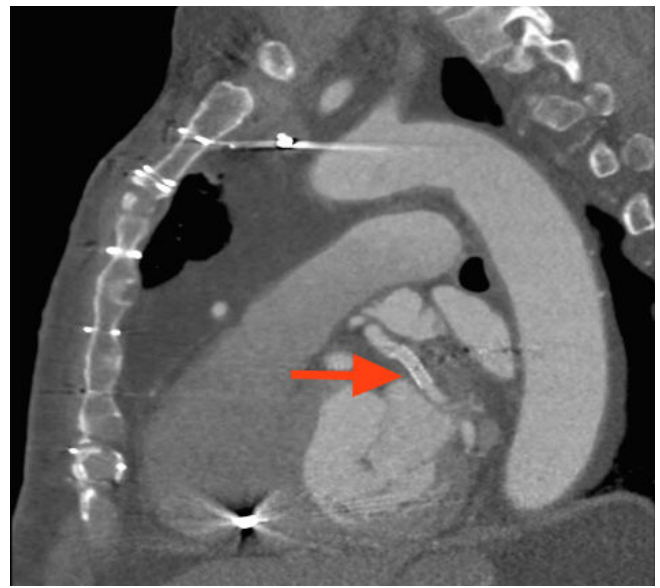

Figure 4 CT angiography at 12 month follow up showing significant decrease in the size of the aneurysm. Covered stent can be seen (red arrow). 
The balloon was pulled back and then the stent was post dilated with a $5.0 \times 12 \mathrm{~mm}$ Quantum apex balloon (Boston Scientific, Natick, MA) at 20 atms. Repeat angiography revealed no flow into the aneurysm with excellent apposition of the Jomed stent. Access site was closed with the Proglide device (Abbott Vascular, Redwood city, CA). The patient was discharged home after one day on dual antiplatelet therapy without any complications. The 12 month CT angiography showed good results (Figure 4).

\section{Discussion}

Coronary artery aneurysms are rare findings detected on coronary angiography. They are either congenital or acquired. Pre-existing CAAs of the native vessels have a propensity to enlarge in size following coronary artery bypass surgery. This is especially common in ectatic arteries [4]. In such situation, surgical resection of the aneurysm might not be an option and percutaneous treatment is a feasible alternative.

The term "Giant" is generally used if the diameter of the coronary artery aneurysm exceeds $8 \mathrm{~mm}$, or is four times larger than the reference vessel. The use of covered stent in the treatment of giant aneurysm has been described [5] However, if an aneurysm has an outflow vessel that can potentially collateralize, excluding the aneurysmal sac using a stent graft might not be sufficient to decrease the risk of rupture. This scenario is analogous to a type 2 endoleak after endovascular repair of abdominal aortic aneursym; and in our opinion requires similar treatment, that is coiling of the potential feeder/collateral vessel antecedent to covered stent deployment [6]. If collateral flow to the aneurysm develops in the future following stent placement, it is virtually impossible to embolize the collaterals.

Coil embolization technique (i.e., multiple coil packing within the sac) provides a physical barrier against incoming flow from the parent artery. The deployed coils reduce the intrasaccular flow rates by generating the form drag and boundary effect induced by the viscosity, promoting intraaneurysmal thrombosis that will ultimately reduce the risk of rupture. In this case, the coils were deployed to embolize the small outflow vessel. This is in contrast to stent assisted coil embolization where coils are utilized only to fill the aneurysmal sac [7]. In addition to the coil deployment, a stent graft can provide another physical barrier to isolate the blood flow from the parent artery to the sac of aneurysm. The balloon expandable stent graft used in this study (i.e., Graftmaster Jostent) was deployed in the neck of aneurysm with a great apposition demonstrating no incoming flow to the sac (confirmed by angiography) after the deployment. The Graftmaster Jostent is the only stent graft that can be used for small diameter less than $4 \mathrm{~mm}$, which is a great candidate device for our treatment option.

Some of the limitations of our study are that no repeat angiography has been done after the procedure, as well as only one case is reported here. The effectiveness and long term outcomes to this technique will have to be studied with serial CT angiography. Primary criteria to assess the outcomes include [1] any potential recanalization with the coil embolization technique along with the regrowth of the sac, [2] intra-aneurysmal thrombosis formation, [3] thrombogenic issue with a stent graft, and [4] any potential luminal area reduction by tissue growth.

\section{References}

1. Crawley PD, Mahlow WJ, Huntsinger DR, Afiniwala S, Wortham DC (2014) Giant coronary artery aneurysms: review and update. Tex Heart Inst J. 41: 603-608.

2. Lazar JF, Compton M, Li F, Knight P (2013) Excising a giant: report of a 7-cm coronary artery aneurysm. Tex Heart Inst J 40: 173-175.

3. Siegrist PT, Sumitsuji S, Osuga K, Sakaguchi T, Tachibana K, et al. (2013) Endovascular coil embolization of Behçet disease-related giant aneurysm of the right coronary artery after failure of surgical suture. JACC Cardiovasc Interv 6: 31-32.

4. Plehn G, Van Bracht M, Zuehlke C, Schrage MO, Wickenbrock I, et al. (2006) From atherosclerotic coronary ectasia to aneurysm: a case report and literature review. Int J Cardiovasc Imaging 22: 311-316.

5. Siregar S, Hoseyni Guyomi S, Van Herwerden LA (2010) Covered stents in giant coronary artery aneurysm. Eur Heart J 31: 2823.

6. Baum RA, Carpenter JP, Stavropoulous SW, Fairman RM (2001) Diagnosis and management of type 2 endoleaks after endovascular aneurysm repair. Tech Vasc Interv Radiol 4: 222-226.

7. Terasawa A, Yokoi T, Kondo K (2013) Stent-assisted coil embolization of coronary artery aneurysm. J Invasive Cardiol 25: 175-177. 